Ann. Génét. Sél. anim., I980, 12 (2), I8I-I89.

\title{
Effet du gène de nanisme lié au sexe dw sur la croissance du poulet : étude biochimique et anatomique
}

\author{
P. VIGNERON, C. BRESSOT et J. GOUSSOPOULOS \\ Station de Physiologie Animale, \\ * Centre de Recherches de Montpellier, I.N.R.A.-E.N.S.A., place Viala, \\ 34060 Montpellier Cedex, France
}

\begin{abstract}
Résumé
L'allométrie de croissance postnatale de l'eau et des lipides corporels totaux a été étudiée chez des poulets porteurs $[d w]$ ou non porteurs $[D w]$ du gène de nanisme. Chez les mâles $[D w]$ nous observons une accumulation isométrique de l'eau ou des lipides, alors que chez les [dw], l'allométrie de croissance est légèrement majorante pour les lipides, minorante pour l'eau. Il en est de même chez les femelles jusqu'au poids vif de I $400 \mathrm{~g}$ chez les $[D w]$ et de $700 \mathrm{~g}$ chez les $[d w]$. Les lipides évoluent par la suite selon une allométrie majorante, l'eau selon une allométrie minorante, avec des changements de pente moins marqués chez les [dw].

Une étude de l'allométrie de croissance des tissus et organes a été menée chez les poules. Chez les $[d w]$, le tibia et le tarsométatarse ont une croissance relative inférieure, et le tissu adipeux dissécable se développe sans changement d'allométrie marqué. Pour les autres tissus ou organes, les pentes des droites d'allométrie ne sont pas significativement différentes entre poules $[D w]$ et $[d w]$.
\end{abstract}

\section{Introduction}

La recherche de poulets de petite taille, d'efficacité supérieure pour la production d'œufs, conduit à sélectionner des souches porteuses du gène de nanisme $d w$. Dans un article de synthèse, GuILLAumE (I976), rappelle les effets de ce gène sur la physiologie, les performances, les besoins alimentaires de tels animaux. Leur croissance et leur composition corporelle ont été également étudiées, mais de façon trop fragmentaire, donnant lieu à la publication de résultats divergents. C'est ainsi que la teneur en lipides corporels est supérieure chez la poulette naine (MERAT et Guillaume, I969, Ricard, I970), mais inférieure chez la poule naine adulte pondeuse (MERAT et RICARD, I964).

Dans ce travail, suite d'une collaboration entreprise entre les stations de recherches avicoles de Nouzilly, du Magneraud, et de la Station de Physiologie animale de Montpellier, nous nous sommes donc attachés à décrire 1'allométrie 
de croissance des tissus et organes ainsi que des constituants biochimiques de poulets porteurs ou non $d u$ gène de nanisme $d w$, notée respectivement $d w d w$ et $D w^{+}$ $d w$ chez les mâles, $d w$ et $\mathrm{D} w^{+}$chez les femelles (MÉRAT et al., I978).

\section{Matériel et méthodes}

Les animaux porteurs du gène $d w$ sont ici également porteurs du gène $k$, emplumement rapide, utilisé comme marqueur. Dans les deux types d'études, anatomique et biochimique, nous avons disposé, à chaque stade et pour chaque sexe, d'une paire d'animaux demi-frères ou demi-sours, l'un des deux exprimant le caractère nain $(d w)$, l'autre non $(D w)$. Tous ces poulets ont été élevés dans les mêmes conditions, recevant ad libitum un aliment granulé du commerce titrant $22,3 \mathrm{p}$. Ioo de protéines et environ I2,75 MJ (3 050 kcal) d'énergie métabolisable par $\mathrm{kg}$.

Les stades d'abattage suivants ont été retenus : I, $2,3,4,5,8$, II, I5, 2I, 34 semaines, afin de faciliter l'étude allométrique. L'analyse de croissance pondérale, effectuée sur les données recueillies par dissection complète a porté sur des poules abattues $\mathrm{I}, 2,3,4,5,8$, II, I5 semaines après l'éclosion.

Les techniques d'analyse chimique (teneur en eau et en lipides) ont été déjà exposées (VIGNERON, I972). L'analyse mathématique fait appel à la relation d'allométrie pour l'étude de l'évolution pondérale des constituants chimiques et des tissus ou organes au cours de la croissance. Cette relation dérive de la fonction puissance $y=a x^{b}$ introduite par HUXIEY (I932) pour décrire la croissance relative d'un organe ou d'une partie $y$ de l'organisme par rapport à $x$, organisme entier ou autre partie de cet organisme. Le coefficient d'allométrie $b$ représente le rapport des vitesses spécifiques de croissancey $\frac{d t}{d y} \int \frac{x d t}{d x}$. Nous avons utilisé la méthode des moindres carrés pour le calcul de la relation d'allométrie rendue linéaire par transformation $\log$ arithmique $\log y=a+b \log x$. Selon que la pente $b$ de la droite est supérieure, égale ou inférieure à $I$, le composant considéré croît plus vite, aussi vite ou moins vite que l'organisme. On dit alors qu'il croît respectivement selon une allométrie majorante, de façon isométrique, ou selon une allométrie minorante. Lorsqu'un changement d'allométrie a été constaté, le poids correspondant au changement de pente a été déduit des équations des droites d'allométrie.

\section{Résultats et discussion}

\section{I. - Croissance pondérale globale:}

L'évolution du poids vif au cours de la croissance est rapportée sur le tableau I, chaque poids représentant la moyenne de 36 individus. Pour les stades $2 \mathrm{r}$ et 34 semaines nous n'avons disposé que de deux individus par points.

Dès la première semaine, le test de comparaison par paires montre que les individus nains $d w$ ont en moyenne une croissance plus lente que les $D w$. BARON (I972) avait d'ailleurs mis à profit la différence de vitesse de croissance spécifique 
TABLEAU I

Croissance pondérale moyenne des poulets nains [dw] ou normaux [Dw] Mean ponderal growth of dwarf [dw] and normal [Dw] chicks

\begin{tabular}{|c|c|c|c|c|c|c|c|c|c|c|c|c|}
\hline \multicolumn{3}{|c|}{$\begin{array}{l}\text { Age } \\
\text { en semaines }\end{array}$} & I & 2 & 3 & 4 & 5 & 8 & I I & I 5 & $2 \mathrm{I}$ & 34 \\
\hline \multirow{3}{*}{ 荧 } & \multirow{2}{*}{$\begin{array}{l}\text { Poids } \\
\text { vif } \\
\text { en } g\end{array}$} & \multirow{2}{*}{$\begin{array}{c}D w \\
d w\end{array}$} & 65,5 & I $3^{8}$ & 224 & $33 \mathrm{I}$ & $47 I$ & I 046 & I 480 & $2 \operatorname{I}_{7} 6$ & \multicolumn{2}{|l|}{$29 \pi 2$} \\
\hline & & & $6 \mathrm{I}$ & 120 & I9I & 268 & 382 & $8 \mathrm{I} 7$ & I I 24 & I 673 & 2347 & \\
\hline & $d w / D w$ & - & 0,93 & 0,87 & 0,85 & $0,8 \mathrm{I}$ & $0,8 \mathrm{I}$ & 0,78 & 0,76 & 0,77 & $0,8 \mathrm{I}$ & \\
\hline \multirow{3}{*}{ 岂 } & \multirow{2}{*}{$\begin{array}{l}\text { Poids } \\
\text { vif } \\
\text { en } g\end{array}$} & \multirow{2}{*}{$\begin{array}{l}D w \\
d w\end{array}$} & 65,5 & I 29 & 211 & 304 & 418 & 9I 8 & I 236 & I $75^{8}$ & $2 \mathrm{I} 30$ & 2995 \\
\hline & & & $6 I$ & I I 8 & I 85 & 253 & 336 & 666 & 896 & I 2 I 6 & I $47 \mathrm{I}$ & I 874 \\
\hline & $d w / D w$ & · & 0,93 & $0,9 \mathrm{I}$ & 0,88 & 0,83 & 0,80 & 0,73 & 0,72 & 0,69 & 0,69 & 0,63 \\
\hline
\end{tabular}

pour la discrimination des poulettes $d w$ et $D w$ dès la deuxième semaine après l'éclosion.

Les poulets décrits ici, bien qu'ayant eu une croissance moins rapide que ceux étudiés par RICARD (I970) aux stades 4, 8 et II semaines expriment des caractéristiques très voisines. La croissance du poulet nain est de plus en plus retardée, et les poules $D w$ ont une croissance plus rapide que les coqs nains. Contrairement aux résultats de HuTr (I959), la réduction de taille des coqs nains semble ici moins importante que celle des poules naines après le stade 4 semaines, le ralentissement de croissance devenant alors plus marqué que chez celles-ci. Le faible nombre d'individus, en particulier dans les derniers stades, n'autorise pas une étude statistique. De plus, quelques animaux présentaient le caractère " cou nu " susceptible d'influencer le métabolisme énergétique (TouchBuRN, I97I).

\section{2. - Évolution de la composition corporelle au cours de la croissance}

Nous avons porté sur le tableau 2 les caractéristiques des droites d'allométrie de croissance des lipides et de l'eau corporelle. Rappelons que pour nous l'eau corporelle comprend l'eau contenue dans le tractus digestif (ingestats, fécès), qui ne représente pas un compartiment distinct dans l'organisme.

Chez les mâles, nous observons une accumulation isométrique des lipides et de l'eau chez les $D w$. Pour les $d w$ l'allométrie de croissance est légèrement majorante pour les lipides, compensée par une allométrie minorante de l'eau.

Chez les femelles, on observe dans les deux cas un phénomène biphasique avec changement d'allométrie vers I $400 \mathrm{~g}$ chez les $[D w], 700 \mathrm{chez}$ les $[d w]$. Avant ces poids, les équations des droites de régression calculées ne sont pas significativement différentes de ce qu'elles sont chez les mâles de même génotype. Les lipides évoluent par la suite selon une allométrie majorante, 1'eau selon une allométrie minorante.

Au cours de cette deuxième phase de développement il est également intéressant de constater que le coefficient d'allométrie des lipides est moins élevé chez les 


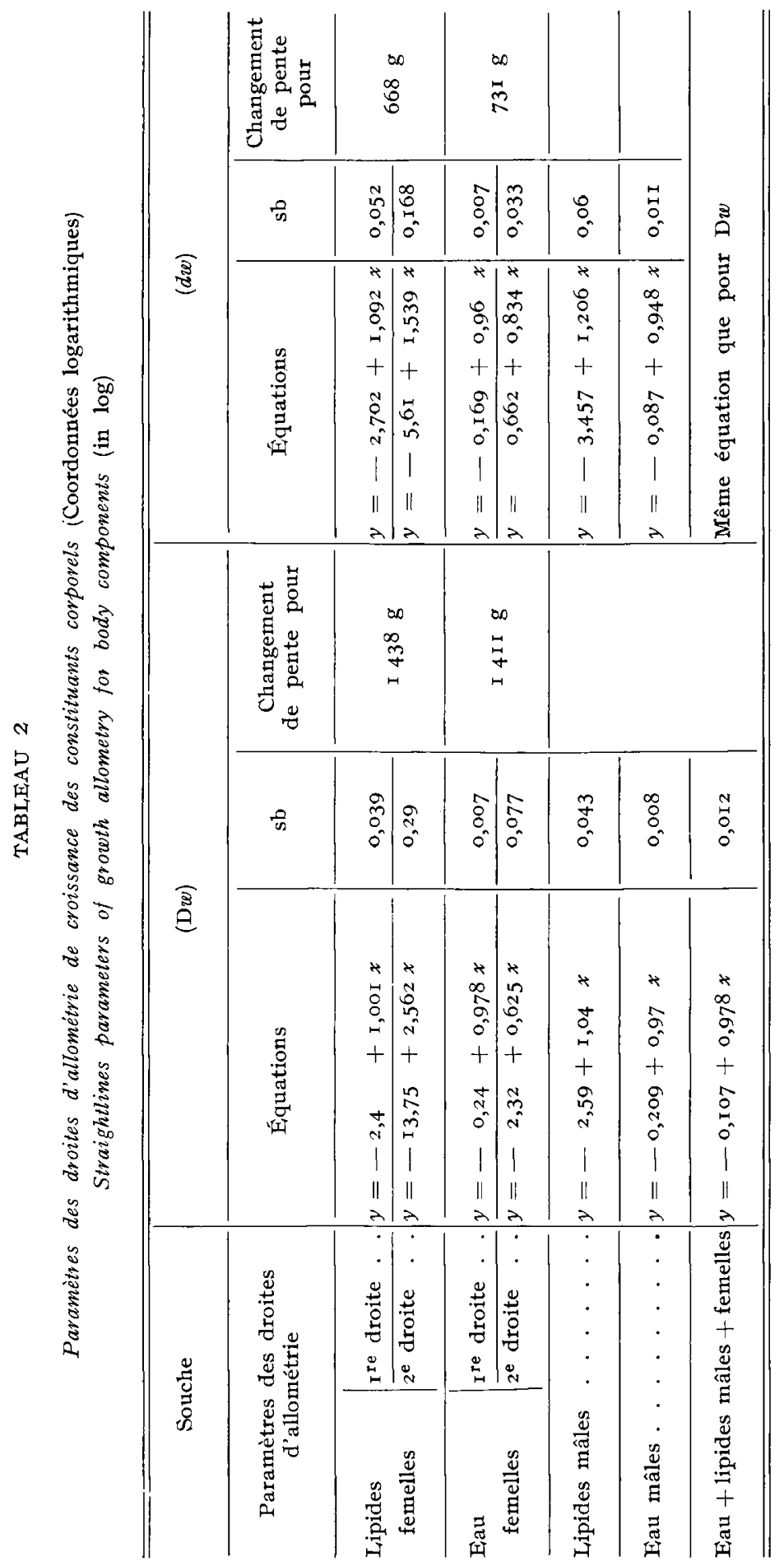


$[d w](b=\mathrm{I}, 53)$ que chez les $[\mathrm{D} w](b=2,562)$ celles-ci accumulant les lipides de façon beaucoup plus active. Il en résulte que les poules $[d w]$ et $[D w]$ présentent la même composition corporelle jusqu'à approximativement $700 \mathrm{~g}$ de poids vif; à poids égal les $[d w]$ sont donc plus riches en lipides à partir de $700 \mathrm{~g}$, et théoriquement jusqu'à $2300 \mathrm{~g}$ dans la mesure où elles peuvent atteindre ce poids, les [Dw] devenant elles-mêmes plus grasses au-delà de ce dernier poids.

Ce phénomène apparaît nettement sur la figure I où l'on a représenté en coordonnées arithmétiques l'accumulation des lipides au cours de la croissance

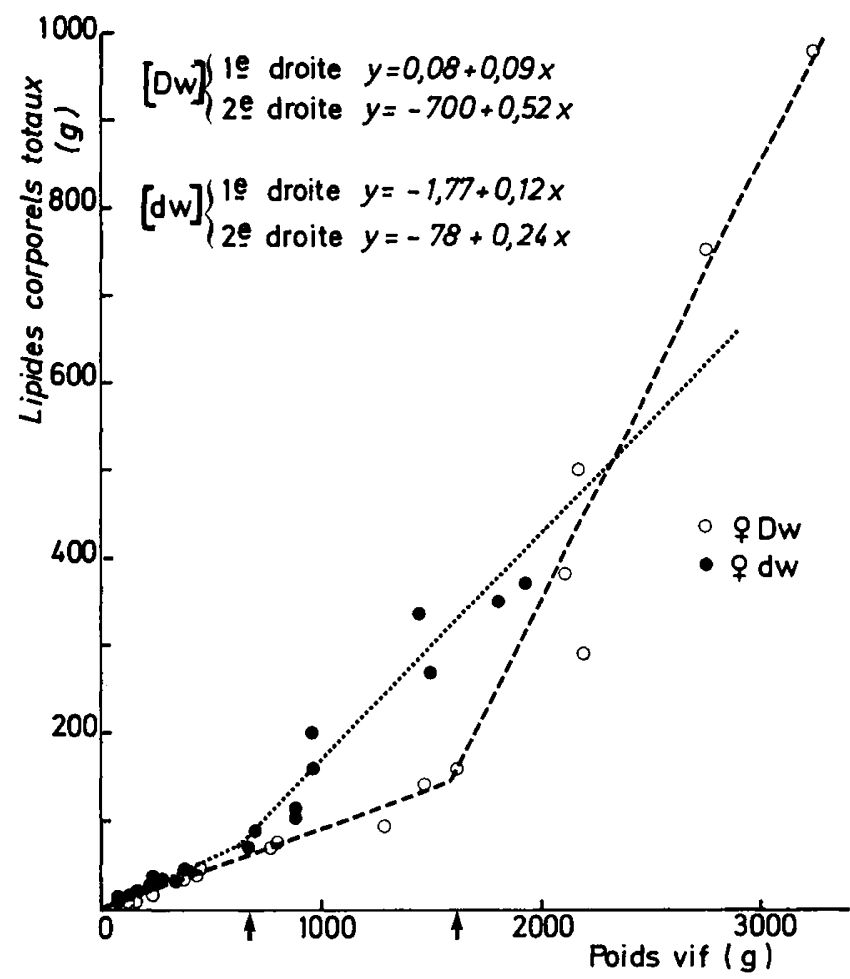

FIG. I. - Evolution postnatale de la quantité de lipiaes corporels chez des poules (Dw) et (dw) Postnatal evolution of the amount of body lipids in $(D w)$ and (dw) hens

chez les poules $[d w]$ et $[D w]$ ainsi que les ajustements linéaires calculés avec ces mêmes données.

La phase d'allométrie majorante des lipides ne semble donc pas liée directement à la ponte qui lui est chronologiguement postérieure. En outre, le début de la ponte est plus tardif chez les poules naines ( 176 jours contre I63) (RICARD, I976).

Les divergences des résultats rapportés dans la littérature quant à la composition corporelle de poules $[d w]$ et $[D w]$ s'expliquent ainsi naturellement par les différences de précocité et de vitesse d'accumulation des lipides et de 1'eau.

L'intérêt de ce phénomène nous a incité à poursuivre cette étude chez les femelles au niveau anatomique. 


\section{3. - Allométrie de croissance des tissus et organes chez les poules}

Les résultats rapportés sur les tableaux 3 et 4 ont été recueillis chez des poules abattues jusqu'au stade I5 semaines inclusivement. Le poids vif vide a été pris comme variable de référence afin de pouvoir comparer les caractéristiques de croissance des poules $D w$ et $d w$ à celles des poules New-Hampshire déjà étudiées par Goussopoulos (I978). Il existe une corrélation de 0,99 entre poids vif vide $y$ et poids vif $x$, la régression linéaire entre ces deux variables, calculée pour les poules $D w$ et $d w$ par la méthode des moindres carrés, étant

$$
y=-3,976+\left(0,95^{8} \pm 0,002\right) x
$$

\section{TABLEAU 3}

Équations des droites d'allométrie calculées pour divers tissus et organes, lorsqu'un changement d'allométrie n'a pas été mis en évidence (coordonnées logarithmiques) Allometric straightlines parameters for tissues and organs developing without change of slope during growth (in log)

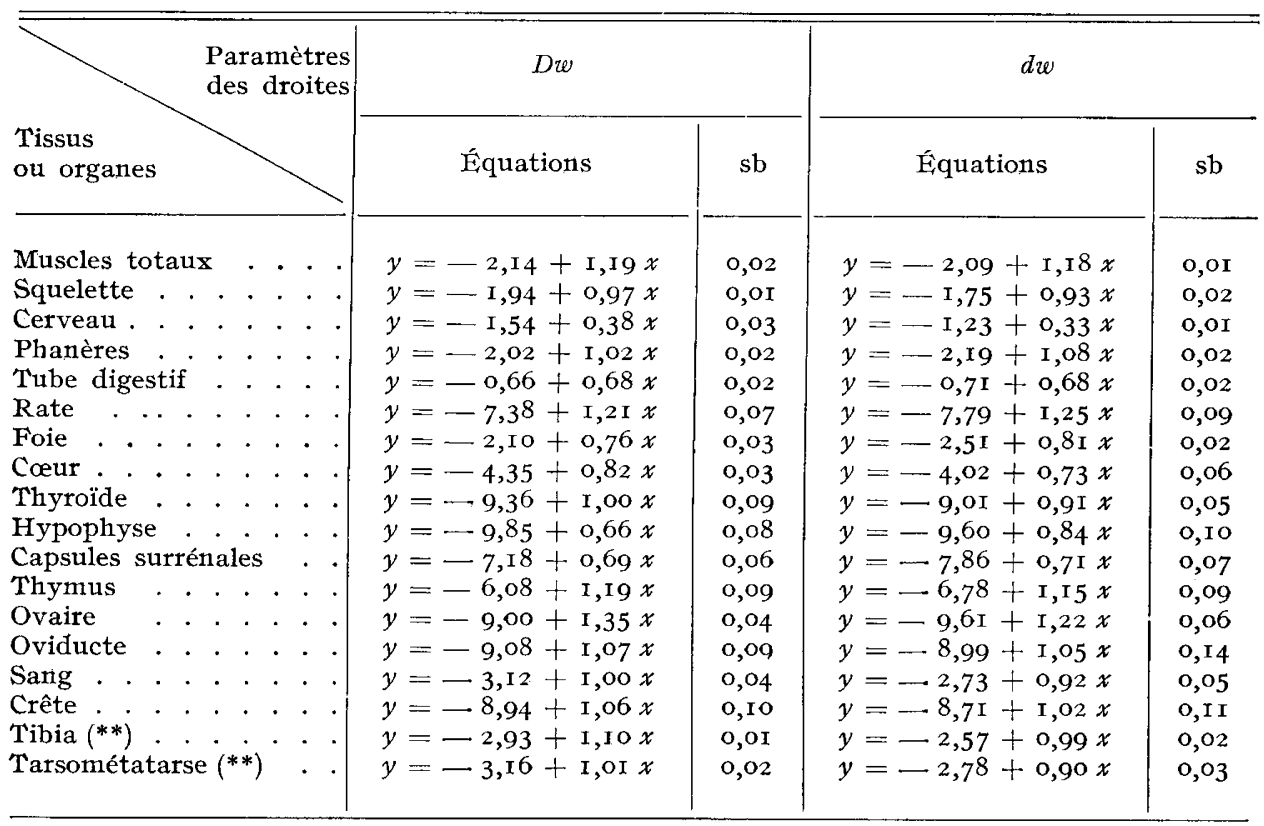

$(* *)$ Différence significative pour $\mathrm{P}<$ o,ox

$(* *)$ significance for $\mathbf{P}<0$,or.

Sur le tableau 3 sont portés les paramètres des droites d'allométrie calculées lorsque ce type d'ajustement peut être retenu pendant la période considérée. Les pentes $b$ de ces droites ne sont pas significativement différentes chez les souches comparées, y con pris pour la thyroïde ( $b$ voisin de $\mathrm{I}$ ), ainsi que pour l'hypophyse et les surrénales ( $b$ voisin de 0,65$)$. Le tibia et le tarsométatarse sont les seuls à 


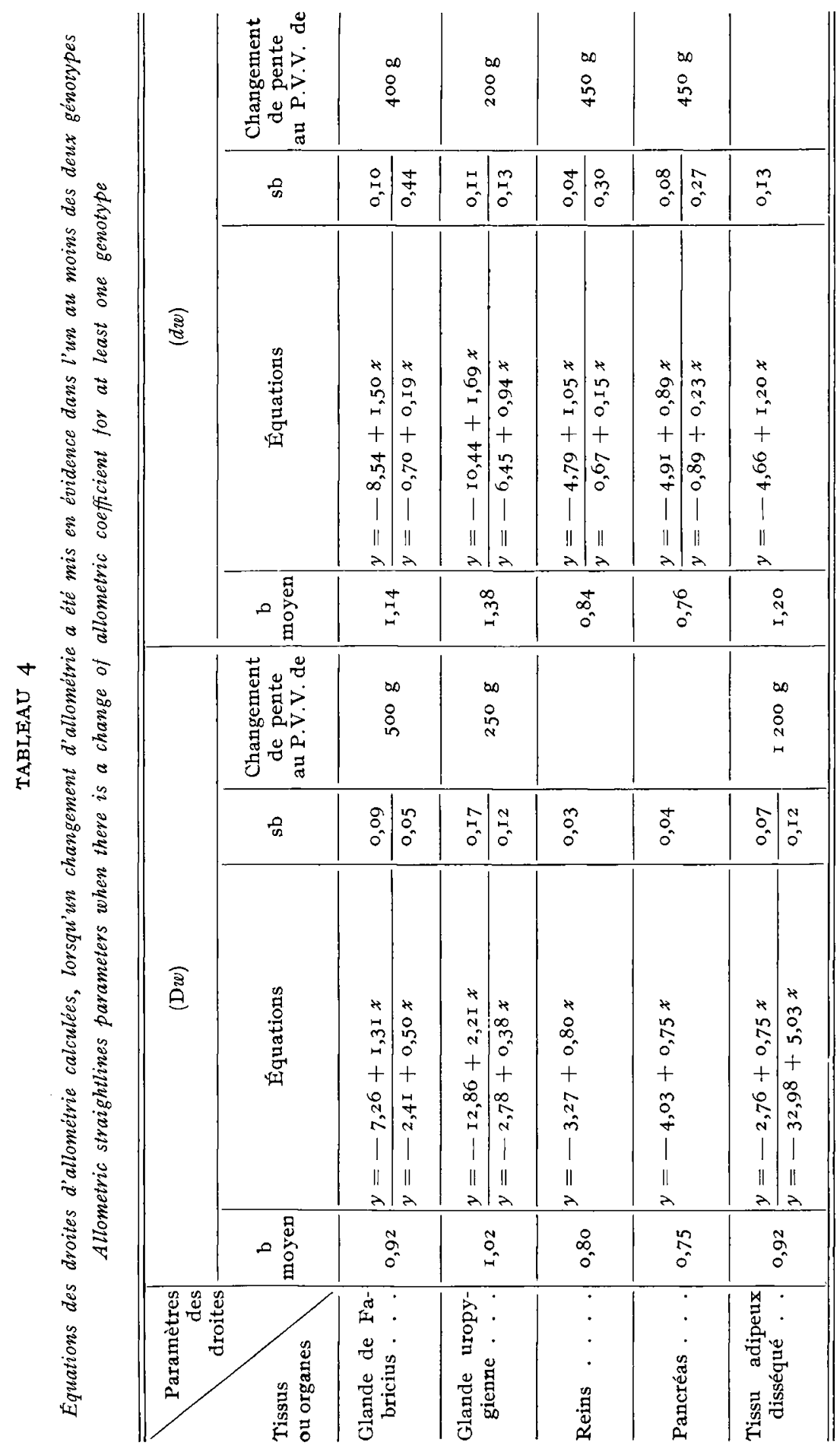


avoir une croissance relative significativement inférieure chez les $d w$, ce qui est une caractéristique constante des animaux nains.

En outre, les pentes indiquées dans le tableau 3 sont voisines ou égales à celles calculées pour les tissus et crganes correspondants chez les poules NewHampshire (Goussopoulos, I978) au cours de la même période postnatale ce qui est en faveur d'une unicité du schéma de développement dans cette espèce malgré la diversité des génotypes.

Sur le tableau 4 se trouvent les paramètres des droites d'allométrie des organes et tissus dont le coefficient d'allométrie varie au cours de la croissance. Le changement d'allométrie n'a pas toujours été mis en évidence dans les deux génotypes.

L'allométrie de la glande uropygienne, très majorante jusqu'au poids vif vide de $200 \mathrm{~g}$ environ, devient minorante par la suite. Cette évolution n'est pas significativement différente pour les poules $[D w],[d w]$ ou New-Hampshire, ce qui est également le cas pour la bourse de Fabricius, le changement d'allométrie survenant alors entre 400 et $500 \mathrm{~g}$ de poids vif.

Pour le pancréas et les reins, dont la croissance relative est très voisine, un changement d'allométrie n'a pas été mis en évidence chez les [Dw], ce qui traduit peut être simplement une précocité plus grande des $[d w]$ à cet égard. Chez la Poule New-Hampshire, étudiée pendant une plus longue période, un ajustement curvilinéaire s'était révélé nécessaire pour ces deux organes.

Le tissu adipeux, qui correspond ici à la masse des dépôts dissécables, présente un net changement d'allométrie vers le poids vif vide de I $200 \mathrm{~g}$ chez les $[D w]$. Le changement d'allométrie des lipides corporels totaux semble donc lié principalement à une mise en réserve accrue des lipides dans les dépôts adipeux.

Chez les $[d w]$, un changement d'allométrie du tissu adipeux n'a pu être mis en évidence. Comme pour les lipides corporels totaux, ce changement, s'il existe, est moins marqué que chez les $[D w]$ et n'a pas été révélé du fait de la variabilité des données.

Ces résultats concordent avec ceux obtenus par RICARD (I976) chez des animaux âgés de I I à 60 semaines, et par MÉRAT et RICARD (I974) chez des poules de plus d'un an, l'état d'engraissement étant estimé par le pourcentage de graisse abdominale. Pour ces auteurs, les différences observées chez les poules adultes proviendraient d'une modification de la physiologie de la formation de l'œuf. Nos résultats montrent clairement qu'il s'agit simplement d'une différence de physiologie du développement.

\section{Conclusion}

Cette étude nous a permis de confirmer et d'expliquer les résultats paradoxaux rapportés dans la littérature quant à l'adiposité de poules naines ou normales. Dans toutes les espèces, le tissu adipeux est le dernier à se développer. Chez les poules naines $[d w]$, la phase active d'accumulation des graisses dans le tissu adipeux débute précocement. Mais dès le stade initial, vers $700 \mathrm{~g}$, alors que la ponte - d'ailleurs tardive chez les $[d w]$ - est loin d'avoir débuté, la vitesse d'accumulation des lipides est inférieure à ce qu'elle sera chez les [Dw] à partir de I $400 \mathrm{~g}$. Les poules naines sont donc plus grasses aux stades jeunes, plus maigres par la suite. Chez les mâles, comme dans d'autres génotypes, nous n'avons pas 
observé de changement d'allométrie des lipides totaux. L'ensemble de ce matériel animal : coqs, poules naines et normales, apparaît donc particulièrement adapté à l'étude des régulations endocriniennes de la lipogenèse.

Reçu pour publication en août 1980.

\title{
Summary
}

\author{
Effects of the sex-linked dwarf gere " $\mathrm{dw}$ " on chick growth: biochemical \\ and anatomical study
}

Postnatal growth allometry of body total water or lipids was studied for brother or sister chicks of dwarf $[d w]$ or normal $[D w]$ genotypes. For $[D w]$ males, isometric accretion of water or lipids occurs throughout growth whereas it is slightly increasing for lipids and slightly decreasing for water in $[d w]$ males.

Similar allometric coefficients are founded in hens till live body weight of $\mathrm{I} 400 \mathrm{~g}$ in [Dw] and $700 \mathrm{~g}$ in $[d w]$. Thereafter lipids accumulate more actively and water less actively, the slope changes of allometric straightlines being less pronounced in $d w$ hens.

Growth allometry of tissues and organs was also determined in hens. It is noticed that in $[d w]$ hens, tibia and tarsometatarse present a lower relative growth and that dissectable adipose depots are developing without allometric changes. For other organs and tissues, allometric coefficients of straightlines are not significantly different between $[d w]$ and $[D w]$.

\section{Références bibliographiques}

BARON R., I972. Description biométrique de la croissance pondérale de poules naines et normales. Discrimination précoce sur le poids corporel. Ann. Genet. Sel. anim. 4, I59-I7I.

Goussopoulos J., I978. Étude de la croissance velative postnatale chez le poulet. Thèse. Doc. Université, I-103, Montpellier.

Guillaume J., I976. The dwarfing gene dw; its application in poultry industry. Wld's Poultry Sci. J., 32, 285-304.

Hut' F. B., I959. Sex linked dwarfism in the fowl. J. Heredity, 50, 209-221.

Huxiey J. S., I932. Problems of relative growth, 276 pages, Methuen, London.

Mérat P., Guillaume J., i 969 . Étude d'un gène de nanisme lié au sexe chez la poule. II. Fonctionnement thyroïdien. Ann. Génét. Sél. anim., 1, I3I-I33.

Mérat P., RICARD F. H., I974. Étude d'un gène de nanisme lié au sexe chez la poule : importance de l'état d'engraissement et gain de poids chez l'adulte. Ann. Genét. Sél. anim. 6, 2 I I-2 I 7.

Mérat P., Stupper, M., COQUerelite G., PERramon A., I978. Sex linked Dwarf gene and chick survival to acute hypoxia. Ann. Genét. Sél. anim., 10, 525-53I.

Ricard F. H., I970. Étude d'un gène de nanisme lié au sexe chez la poule. IV. Observations sur la croissance et les caractéristiques de carcasse du jeune poulet. Ann. Genét. Sél. anim., 2, I9-3r.

RICARD F. H., I976. Bases scientifiques de l'utilisation du gène de nanisme dw dans la production $d u$ poulet de chair. Vth European Poultry Conference. Malta.

Touchburn S. P., I97I. Étude de la lipogenèse à l'aide d'un acétate marqué au ${ }^{14} \mathrm{C}$ chez des paires de sœurs normales et naines. Ann. Genét. Sél. anim., 3, 386.

VIGNERON P., I972. Évolution postnatale de la composition corporelle globale chez le poulet. C.R. Soc. Biol., 166, I I 46-I I 49 . 